\title{
Effect of phase inversion of radiofrequency pulses on the recoupling of anisotropic NMR interactions under magic-angle spinning
}

\author{
Fang-Chieh Chou, Shing-Jong Huang, Jerry C.C. Chan* \\ Department of Chemistry, National Taiwan University, No. 1, Section 4, Roosevelt Road, Taipei, Taiwan
}

\section{A R T I C L E I N F O}

\section{Article history:}

Received 3 December 2008

In final form 16 January 2009

Available online 23 January 2009

\begin{abstract}
A B S T R A C T
The effect of phase inversion of radiofrequency pulses on the recoupling of anisotropic chemical shift and heteronuclear magnetic dipole-dipole interactions has been investigated by a simple analytical treatment. For the recoupling of chemical shift anisotropy, the evolution of the spin system will be different if the sign of all rf phases is inverted. This phenomenon is readily explained by the higher-order effect of average Hamiltonian theory. On the contrary, such dramatic phase inversion effect is not observed for heteronuclear dipolar recoupling, which can also be rationalized in our treatment. Numerical simulations are carried out to corroborate our analytical results and experimental verification is obtained for $\left[1-{ }^{13} \mathrm{C}\right]$-alanine.

(c) 2009 Published by Elsevier B.V.
\end{abstract}

\section{Introduction}

Solid-state nuclear magnetic resonance (SSNMR) has proven to be a powerful technique for the structural elucidation of biological solids [1]. The techniques used to restore the anisotropic interactions under the high-resolution condition provided by magic-angle spinning (MAS) are generally known as recoupling [2,3]. Recently, Levitt and co-workers have developed a splendid collection of symmetry-based pulse sequences, which have provided a very general theoretical framework for the discussion of rotor synchronized recoupling pulse sequences in SSNMR spectroscopy [4,5]. In particular, $C N_{n}^{v}$ pulse sequences comprise $N$ repetitions of a subcycle $C$, spanning a total of $n$ rotor periods $\left(n \tau_{R}\right)[4,6,7] . C$ is a radiofrequency (rf) pulse sequence of length $\tau_{C}=n \tau_{R} / N$, comprising of pulses that the net rotation in each subcycle is an integer multiple of $2 \pi$. An overall rf phase shift $2 \pi q v / N$ is applied to the $q$ th repetition of $C$, where $q$ is an integer running from 0 to $N-1$. By changing the $v$ of $\mathrm{CN}_{n}^{v}$ to $-v$, the sign of all rf phases are inverted. This operation is equivalent to a reflection of the excitation field in the $x z$-plane [5], which may cause a sign change of the first-order average Hamiltonian, depending on the parity of the spin interaction under consideration [8]. This property has been exploited for the design of supercycle to suppress undesirable higher order effects in $\mathrm{CN}_{n}^{v}$ sequences [4].

In this work, we show that one may need to be cautious to the correspondence between spin-dynamics phases and pulse program phases of NMR spectrometers [9-11] for the data analyses of chemical shift recoupling experiments, because of the effect of phase inversion on the spin dynamics. As an experimental verification, measurements were carried out for $\left[1-{ }^{13} \mathrm{C}\right]$-L-alanine. On the

\footnotetext{
* Corresponding author. Fax: +88622363 6359.

E-mail address: chanjcc@ntu.edu.tw (J.C.C. Chan).
}

other hand, we find that such sign inversion of the rf pulses is of no significant consequence for heteronuclear dipolar recoupling up to the second-order, in stark contrast to the case of chemical shift interaction. Although we focus on the $\mathrm{CN}_{n}^{v}$ and $\mathrm{R} N_{n}^{v}$ sequences in our treatment, our results are generally valid for any rotor-synchronized recoupling pulse sequences.

\section{Theory}

\subsection{Chemical shift anisotropy (CSA)}

Under the condition of magic angle spinning (MAS), the Hamiltonian of an isolated spin $1 / 2$ system can be expressed in the following form (for simplicity we ignore the terms of isotropic chemical shift and resonance offset):

$H(t)=H_{\mathrm{rf}}(t)+H_{\mathrm{CSA}}(t)$,

$H_{\mathrm{CSA}}(t)=\omega_{\mathrm{CSA}}(t) I_{z}$

In the interaction frame defined by an rf Hamiltonian of the following form:

$H_{\mathrm{rf}}(t)=\omega_{x}(t) I_{x}+\omega_{y}(t) I_{y}$

the anisotropic chemical shift Hamiltonian becomes

$H_{\mathrm{CSA}}^{T}(t)=\omega_{\mathrm{CSA}}(t) U_{\mathrm{rf}}^{-1} I_{z} U_{\mathrm{rf}}$,

$U_{\mathrm{rf}}=\widehat{T} \exp \left[-i \int_{0}^{t}\left(\omega_{x}\left(t^{\prime}\right) I_{x}+\omega_{y}\left(t^{\prime}\right) I_{y}\right) d t^{\prime}\right]$.

When we change the sign of all phases of the applied rf pulses, the rf Hamiltonian becomes

$H_{r f-}(t)=\omega_{x}(t) I_{x}-\omega_{y}(t) I_{y}$

and we hence obtain for the phase inversion case the following results: 
$H_{\mathrm{CSA}-}^{T}(t)=\omega_{\mathrm{CSA}}(t) U_{\mathrm{rf}-}^{-1} I_{z} U_{\mathrm{rf}-}$,

$U_{\mathrm{rf}-}=\widehat{T} \exp \left[-i \int_{0}^{t}\left(\omega_{x}\left(t^{\prime}\right) I_{x}-\omega_{y}\left(t^{\prime}\right) I_{y}\right) d t^{\prime}\right]$.

The unitary operator $U_{\mathrm{rf}}$ and its phase inversion analog are related by a $\pi$ rotation about the $x$ axis, denoted by $R=\exp \left(-i \pi I_{x}\right)$ :

$U_{\mathrm{rf}-}=R^{-1} U_{\mathrm{rf}} R$

and

$U_{\mathrm{rf}-}^{-1}=\left(R^{-1} U_{\mathrm{rf}} R\right)^{-1}=R^{-1} U_{\mathrm{rf}}^{-1} R$.

Consequently, $H_{\mathrm{CSA}-}^{T}$ and $H_{\mathrm{CSA}}^{T}$ have the following relationship:

$\begin{aligned} H_{\mathrm{CSA}-}^{T}(t) & =\omega_{\mathrm{CSA}}(t) U_{\mathrm{rf}-}^{-1} I_{z} U_{\mathrm{rf}-}=\omega_{\mathrm{CSA}}(t) R^{-1} U_{\mathrm{rf}}^{-1} R I_{z} R^{-1} U_{\mathrm{rf}} R \\ & =-\omega_{\mathrm{CSA}}(t) R^{-1} U_{\mathrm{f}}^{-1} I_{z} U_{\mathrm{rf}} R=-R^{-1} H_{\mathrm{CSA}}^{T}(t) R .\end{aligned}$

In general, the chemical shift Hamiltonian in the interaction frame has the following form:

$H_{\mathrm{CSA}}^{T}(t)=a(t) I_{x}+b(t) I_{y}+c(t) I_{z}$

Based on the above result, we obtain

$H_{\mathrm{CSA}-}^{T}(t)=-R^{-1} H_{\mathrm{CSA}}^{T} R=-a(t) I_{x}+b(t) I_{y}+c(t) I_{z}$.

Using the Magnus expansion, the lowest-order average Hamiltonian over a time period of $t_{c}$ can be expressed as

$\begin{aligned} \bar{H}_{\mathrm{CSA}}^{(1)} & =\frac{1}{t_{c}} \int_{0}^{t_{c}} a\left(t^{\prime}\right) I_{x} d t^{\prime}+\frac{1}{t_{c}} \int_{0}^{t_{c}} b\left(t^{\prime}\right) I_{y} d t^{\prime}+\frac{1}{t_{c}} \int_{0}^{t_{c}} c\left(t^{\prime}\right) I_{z} d t^{\prime} \\ & \equiv a_{1} I_{x}+b_{1} I_{y}+c_{1} I_{z},\end{aligned}$

where the magnitudes of the constants $a_{1}, b_{1}$, and $c_{1}$ depend on the particulars of the rf pulse sequence. Similarly, we have

$\bar{H}_{\mathrm{CSA}-}^{(1)}=-a_{1} I_{x}+b_{1} I_{y}+c_{1} I_{z}$.

The corresponding second-order Hamiltonians are calculated as

$$
\begin{aligned}
\bar{H}_{\mathrm{CSA}}^{(2)}= & \frac{-i}{2 t_{c}} \int_{0}^{t_{c}} d t \int_{0}^{t} d t^{\prime} a(t) b\left(t^{\prime}\right)\left[I_{x}, I_{y}\right] \\
& +\frac{-i}{2 t_{c}} \int_{0}^{t_{c}} d t \int_{0}^{t} d t^{\prime} b(t) a\left(t^{\prime}\right)\left[I_{y}, I_{x}\right] \\
& +\frac{-i}{2 t_{c}} \int_{0}^{t_{c}} d t \int_{0}^{t} d t^{\prime} a(t) c\left(t^{\prime}\right)\left[I_{x}, I_{z}\right] \\
& +\frac{-i}{2 t_{c}} \int_{0}^{t_{c}} d t \int_{0}^{t} d t^{\prime} c(t) a\left(t^{\prime}\right)\left[I_{z}, I_{x}\right] \\
& +\frac{-i}{2 t_{c}} \int_{0}^{t_{c}} d t \int_{0}^{t} d t^{\prime} b(t) c\left(t^{\prime}\right)\left[I_{y}, I_{z}\right] \\
& +\frac{-i}{2 t_{c}} \int_{0}^{t_{c}} d t \int_{0}^{t} d t^{\prime} c(t) b\left(t^{\prime}\right)\left[I_{z}, I_{y}\right] \\
& \equiv a_{2} I_{x}+b_{2} I_{y}+c_{2} I_{z} .
\end{aligned}
$$

Similarly, we obtain for the phase inversion case the following results:

$$
\begin{aligned}
\bar{H}_{\mathrm{CSA}-}^{(2)} & =-\frac{-i}{2 t_{c}} \int_{0}^{t_{c}} d t \int_{0}^{t} d t^{\prime} a(t) b\left(t^{\prime}\right)\left[I_{x}, I_{y}\right] \\
& -\frac{-i}{2 t_{c}} \int_{0}^{t_{c}} d t \int_{0}^{t} d t^{\prime} b(t) a\left(t^{\prime}\right)\left[I_{y}, I_{x}\right] \\
& -\frac{-i}{2 t_{c}} \int_{0}^{t_{c}} d t \int_{0}^{t} d t^{\prime} a(t) c\left(t^{\prime}\right)\left[I_{x}, I_{z}\right] \\
& -\frac{-i}{2 t_{c}} \int_{0}^{t_{c}} d t \int_{0}^{t} d t^{\prime} c(t) a\left(t^{\prime}\right)\left[I_{z}, I_{x}\right] \\
& +\frac{-i}{2 t_{c}} \int_{0}^{t_{c}} d t \int_{0}^{t} d t^{\prime} b(t) c\left(t^{\prime}\right)\left[I_{y}, I_{z}\right] \\
& +\frac{-i}{2 t_{c}} \int_{0}^{t_{c}} d t \int_{0}^{t} d t^{\prime} c(t) b\left(t^{\prime}\right)\left[I_{z}, I_{y}\right]=a_{2} I_{x}-b_{2} I_{y}-c_{2} I_{z} .
\end{aligned}
$$

When $\bar{H}_{\mathrm{CSA}}^{(1)}$ is non-gamma encoded, i.e. $a_{1}=b_{1}=0$, we have

$\bar{H}_{\mathrm{CSA}}^{(1)}=\bar{H}_{\mathrm{CSA}-}^{(1)}=c_{1} I_{z}$

Presumably all the second-order terms not commuting with $I_{z}$ will be truncated and hence we obtain the overall average Hamiltonian up to the second-order term as

$\bar{H}_{\mathrm{CSA}} \cong\left(c_{1}+c_{2}\right) I_{z}$,

$\bar{H}_{\mathrm{CSA}-} \cong\left(c_{1}-c_{2}\right) I_{z}$.

It is noteworthy that the phase inversion of all the rf pulses will affect the spin dynamics if the second-order effects are significant.

On the other hand, when $\bar{H}_{\mathrm{CSA}}^{(1)}$ is gamma encoded, i.e. $c_{1}=0$, we have

$\bar{H}_{\mathrm{CSA}}^{(1)}=a_{1} I_{x}+b_{1} I_{y}$,

$\bar{H}_{\mathrm{CSA}-}^{(1)}=-a_{1} I_{x}+b_{1} I_{y}$.

Note that the evolution of the spin system described by $\bar{H}_{\mathrm{CSA}}^{(1)}$ and $\bar{H}_{\text {CSA- }}^{(1)}$ is identical when the initial density operator is proportional to $I_{z}$. Nevertheless, when the second-order terms containing the transverse spin operators are included, we obtain

$\bar{H}_{\mathrm{CSA}} \cong \bar{H}_{\mathrm{CSA}}^{(1)}+\bar{H}_{\mathrm{CSA}}^{(2)}=\left(a_{1}+a_{2}\right) I_{x}+\left(b_{1}+b_{2}\right) I_{y}$,

$\bar{H}_{\mathrm{CSA}-} \cong \bar{H}_{\mathrm{CSA}-}^{(1)}+\bar{H}_{\mathrm{CSA}-}^{(2)}=\left(-a_{1}+a_{2}\right) I_{x}+\left(b_{1}-b_{2}\right) I_{y}$.

Because the magnitudes of $\left|\bar{H}_{\mathrm{CSA}}\right|$ and $\left|\bar{H}_{\mathrm{CSA}-}\right|$ are different, it can be concluded again that the phase inversion of all the rf pulses will affect the spin dynamics if the second-order effects are significant.

\subsection{Heteronuclear dipole-dipole interaction}

Consider an isolated spin-pair of the heteronuclei $I$ and $S$. Following the procedure described above, the heteronuclear dipolar Hamiltonians in the interaction frame transformed by the rf sequences described in Eqs. (3) and (6) are calculated as

$H_{I S}^{T}(t)=a(t) I_{x} S_{z}+b(t) I_{y} S_{z}+c(t) I_{z} S_{z}$,

$H_{I S_{-}}^{T}(t)=-a(t) I_{x} S_{z}+b(t) I_{y} S_{z}+c(t) I_{z} S_{z}$.

Using the Magnus expansion, the lowest-order Hamiltonians are written as:

$\bar{H}_{I S}^{(1)}=a_{1} I_{x} S_{z}+b_{1} I_{y} S_{z}+c_{1} I_{z} S_{z}$,

$\bar{H}_{I S-}^{(1)}=-a_{1} I_{x} S_{z}+b_{1} I_{y} S_{z}+c_{1} I_{z} S_{z}$

and the second-order Hamiltonians are obtained as

$$
\begin{aligned}
\bar{H}_{I S}^{(2)} & =\frac{-i}{2 t_{c}} \int_{0}^{t_{c}} d t \int_{0}^{t} d t^{\prime}\left[H^{T}(t), H^{T}\left(t^{\prime}\right)\right] \\
& =\frac{-i}{2 t_{c}} \int_{0}^{t_{c}} d t \int_{0}^{t} d t^{\prime} a(t) b\left(t^{\prime}\right)\left[I_{x} S_{z}, I_{y} S_{z}\right] \\
& +\frac{-i}{2 t_{c}} \int_{0}^{t_{c}} d t \int_{0}^{t} d t^{\prime} b(t) a\left(t^{\prime}\right)\left[I_{y} S_{z}, I_{x} S_{z}\right] \\
& +\frac{-i}{2 t_{c}} \int_{0}^{t_{c}} d t \int_{0}^{t} d t^{\prime} a(t) c\left(t^{\prime}\right)\left[I_{x} S_{z}, I_{z} S_{z}\right] \\
& +\frac{-i}{2 t_{c}} \int_{0}^{t_{c}} d t \int_{0}^{t} d t^{\prime} c(t) a\left(t^{\prime}\right)\left[I_{z} S_{z}, I_{x} S_{z}\right] \\
& +\frac{-i}{2 t_{c}} \int_{0}^{t_{c}} d t \int_{0}^{t} d t^{\prime} b(t) c\left(t^{\prime}\right)\left[I_{y} S_{z}, I_{z} S_{z}\right] \\
& +\frac{-i}{2 t_{c}} \int_{0}^{t_{c}} d t \int_{0}^{t} d t^{\prime} c(t) b\left(t^{\prime}\right)\left[I_{z} S_{z}, I_{y} S_{z}\right] \\
& =a_{2} I_{x}+b_{2} I_{y}+c_{2} I_{z},
\end{aligned}
$$


$\bar{H}_{I S-}^{(2)}=a_{2} I_{x}-b_{2} I_{y}-c_{2} I_{z}$.

Hence, the average Hamiltonians up to the second-order terms are

$\bar{H}_{I S} \cong \bar{H}_{I S}^{(1)}+\bar{H}_{I S}^{(2)}=a_{1} I_{x} S_{z}+b_{1} I_{y} S_{z}+c_{1} I_{z} S_{z}+a_{2} I_{x}+b_{2} I_{y}+c_{2} I_{z}$,

$\bar{H}_{I S-} \cong \bar{H}_{I S-}^{(1)}+\bar{H}_{I S-}^{(2)}=-a_{1} I_{x} S_{z}+b_{1} I_{y} S_{z}+c_{1} I_{z} S_{z}+a_{2} I_{x}-b_{2} I_{y}-c_{2} I_{z}$.

For the non-gamma encoded case, i.e. $a_{1}=b_{1}=0$, Eqs. (31) and (32) are simplified to read

$\bar{H}_{I S} \cong c_{1} I_{z} S_{z}+c_{2} I_{z}$

$\bar{H}_{I S-} \cong c_{1} I_{z} S_{z}-c_{2} I_{z}$,

where we assume that the first-order terms will truncate those second-order terms which do not commute with $I_{z}$. If the initial density operator is proportional to $I_{x}$, the evolution of the system is calculated as

$$
\begin{aligned}
\rho\left(t_{c}\right) & =\exp \left(-i \bar{H}_{I S} t_{c}\right) I_{x} \exp \left(i \bar{H}_{I S} t_{c}\right) \\
& =\exp \left(-i c_{1} I_{z} S_{z} t_{c}\right) \exp \left(-i c_{2} I_{z} t_{c}\right) I_{x} \exp \left(i c_{2} I_{z} t_{c}\right) \exp \left(i c_{1} I_{z} S_{z} t_{c}\right) \\
& =\cos \left(c_{1} t_{c}\right) \cos \left(c_{2} t_{c}\right) I_{x}+\cos \left(c_{1} t_{c}\right) \sin \left(c_{2} t_{c}\right) I_{y}+\cdots
\end{aligned}
$$

and

$$
\begin{aligned}
\rho-\left(t_{c}\right) & =\exp \left(-i \bar{H}_{I S-} t_{c}\right) I_{x} \exp \left(i \bar{H}_{I S-} t_{c}\right) \\
& =\exp \left(-i c_{1} I_{z} S_{z} t_{c}\right) \exp \left(i c_{2} I_{z} t_{c}\right) I_{x} \exp \left(-i c_{2} I_{z} t_{c}\right) \exp \left(i c_{1} I_{z} S_{z} t_{c}\right) \\
& =\cos \left(c_{1} t_{c}\right) \cos \left(c_{2} t_{c}\right) I_{x}-\cos \left(c_{1} t_{c}\right) \sin \left(c_{2} t_{c}\right) I_{y}+\cdots
\end{aligned}
$$

for the phase inversion case. Other multiple-quantum terms are dropped in Eqs. (35) and (36). In stark contrast to the case of CSA, the phase inversion of the rf pulses would not cause any appreciable difference in the $x$-component of the magnetization. Consider the gamma encoded case, i.e. $c_{1}=0$, Eqs. (31) and (32) are simplified to read:

$\bar{H}_{I S} \cong \bar{H}_{I S}^{(1)}+\bar{H}_{I S}^{(2)}=a_{1} I_{x} S_{z}+b_{1} I_{y} S_{z}+a_{2} I_{x}+b_{2} I_{y}+c_{2} I_{z}$,

$\bar{H}_{I S-} \cong \bar{H}_{I S-}^{(1)}+\bar{H}_{I S-}^{(2)}=-a_{1} I_{x} S_{z}+b_{1} I_{y} S_{z}+a_{2} I_{x}-b_{2} I_{y}-c_{2} I_{z}$.

In general, the first- and second-order terms of Eqs. (37) and (38) do not commute. For convenience, we define the projection of $a_{2} I_{x}+$ $b_{2} I_{y}+c_{2} I_{z}$ onto $a_{1} I_{x}+b_{1} I_{y}$ as $\left\{\left(a_{1} a_{2}+b_{1} b_{2}\right) / \sqrt{a_{1}^{2}+b_{1}^{2}}\right\}\left(a_{1} I_{x}+b_{1} I_{y}\right)$, which does commute with the first-order term of Eq. (37). For Eq. (38), we can define a similar quantity. Consequently, after incorporating the truncation effect by the first-order terms, Eqs. (37) and (38) become

$\bar{H}_{I S}=a_{1} I_{x} S_{z}+b_{1} I_{y} S_{z}+p\left(a_{1} I_{x}+b_{1} I_{y}\right)$,
$\bar{H}_{I S-}=-a_{1} I_{x} S_{z}+b_{1} I_{y} S_{z}-p\left(-a_{1} I_{x}+b_{1} I_{y}\right)$,

where

$p \equiv\left(a_{1} a_{2}+b_{1} b_{2}\right) / \sqrt{a_{1}^{2}+b_{1}^{2}}$.

Accordingly, the time evolution operator can be expressed as

$$
\begin{aligned}
U_{I S} & =\exp \left(-i \bar{H}_{I S} t_{c}\right) \\
& =\exp \left\{-i\left[\left(a_{1} I_{x} S_{z}+b_{1} I_{y} S_{z}\right)+p\left(a_{1} I_{x}+b_{1} I_{y}\right)\right] t_{c}\right\} \\
& =\exp \left[-i\left(a_{1} I_{x} S_{z}+b_{1} I_{y} S_{z}\right) t_{c}\right] \exp \left[-i p\left(a_{1} I_{x}+b_{1} I_{y}\right) t_{c}\right],
\end{aligned}
$$

$$
\begin{aligned}
U_{I S-} & =\exp \left(-i \bar{H}_{I S-} t_{c}\right) \\
& =\exp \left\{-i\left[\left(-a_{1} I_{x} S_{z}+b_{1} I_{y} S_{z}\right)-p\left(-a_{1} I_{x}+b_{1} I_{y}\right)\right] t_{c}\right\} \\
& =\exp \left[-i\left(-a_{1} I_{x} S_{z}+b_{1} I_{y} S_{z}\right) t_{c}\right] \exp \left[i p\left(-a_{1} I_{x}+b_{1} I_{y}\right) t_{c}\right]
\end{aligned}
$$

because $\left[a_{1} I_{x} S_{z}+b_{1} I_{y} S_{z}, a_{1} I_{x}+b_{1} I_{y}\right]=0$ and $\left[-a_{1} I_{x} S_{z}+b_{1} I_{y} S_{z},-a_{1} I_{x}+\right.$ $\left.b_{1} I_{y}\right]=0$. If the initial density operator is proportional to $I_{z}$, based on Eq. (42) the evolution of the system is calculated as

$$
\begin{aligned}
\rho\left(t_{c}\right)= & U_{I S} I_{z} U_{I S}^{-1} \\
= & \exp \left[-i\left(a_{1} I_{x} S_{z}+b_{1} I_{y} S_{z}\right) t_{c}\right] \exp \left[-i p\left(a_{1} I_{x}+b_{1} I_{y}\right) t_{c}\right] I_{z} \\
& \times \exp \left[i p\left(a_{1} I_{x}+b_{1} I_{y}\right) t_{c}\right] \exp \left[i\left(a_{1} I_{x} S_{z}+b_{1} I_{y} S_{z}\right) t_{c}\right] \\
= & \cos \left(\sqrt{a_{1}^{2}+b_{1}^{2}} t_{c}\right) \cos \left(p \sqrt{a_{1}^{2}+b_{1}^{2}} t_{c}\right) I_{z}+\cdots
\end{aligned}
$$

For the phase inversion case, we obtain

$$
\begin{aligned}
\rho_{-}\left(t_{c}\right)= & U_{I S-} I_{z} U_{I S-}^{-1}=\exp \left[-i\left(-a_{1} I_{x} S_{z}+b_{1} I_{y} S_{z}\right) t_{c}\right] \\
& \times \exp \left[i p\left(-a_{1} I_{x}+b_{1} I_{y}\right) t_{c}\right] I_{z} \exp \left[i\left(-a_{1} I_{x} S_{z}+b_{1} I_{y} S_{z}\right) t_{c}\right] \\
& \times \exp \left[-i p\left(-a_{1} I_{x}+b_{1} I_{y}\right) t_{c}\right]=\cos \left(\sqrt{a_{1}^{2}+b_{1}^{2}} t_{c}\right) \\
& \times \cos \left(p \sqrt{a_{1}^{2}+b_{1}^{2}} t_{c}\right) I_{z}+\cdots
\end{aligned}
$$

The result is similar to the non-gamma encoded case: the phase inversion of the rf pulses would not cause any appreciable difference in the $z$-component of the magnetization.

\section{Experimental methods}

All NMR experiments were carried out at ${ }^{13} \mathrm{C}$ and ${ }^{1} \mathrm{H}$ frequencies of $75.5 \mathrm{MHz}$ and $300.1 \mathrm{MHz}$, respectively, on a Bruker DSX300 NMR spectrometer equipped with a commercial $2.5 \mathrm{~mm}$ triple-resonance probe. The magic-angle spinning (MAS) frequency was set to $10 \mathrm{kHz}$ and its variation was limited to $\pm 4 \mathrm{~Hz}$ using a commercial pneumatic control unit (Bruker, MAS II). The sample was confined in the middle one-half of the rotor volume using Teflon spacers. Typically, during the cross-polarization contact time (1.5 ms), the ${ }^{1} \mathrm{H}$ nutation frequency was set to $50 \mathrm{kHz}$ and that of ${ }^{13} \mathrm{C}$ was ramped through the Hartmann-Hahn matching condition $[12,13]$. Continuous-wave and XiX [14] proton decouplings of $120 \mathrm{kHz}$ were applied during the recoupling and the $t_{2}$ acquisition periods, respectively. For the pulse symmetry of $C 4_{3}^{1}$, the POST composite pulse is incorporated into each subcycle [15]. During the $C 4_{3}^{1}$ recoupling period, the ${ }^{13} \mathrm{C}$ nutation frequency was set to $26.7 \mathrm{kHz}$.

Numerical simulations were carried out using the package SIMPSON (version 1.1.2) [16]. The maximum time step over which the Hamiltonian was approximated to be time independent was set to $0.1 \%$ of the rotor period. Typically, a powder averaging scheme containing 320 REPULSION angles ( $\alpha$ and $\beta$ ) [17] and 36 $\gamma$ angles was chosen. Relaxation effects were ignored.

\section{Results and discussion}

\subsection{Numerical simulations}

To verify the phase inversion effect, we have carried out simulations based on two pulse symmetries, viz. $R 12_{9}^{1}$ and $C 4_{3}^{1}$, which can be used to recouple the chemical shift interaction or heteronuclear dipole-dipole interaction. The $R 12_{9}^{1}$ sequence is non-gamma encoded, whereas $C 4_{3}^{1}$ is gamma encoded. Fig. 1 shows the simulations obtained for the CSA recoupling at two different spinning frequencies. As predicted by our earlier analysis, both gammaencoded and non-gamma-encoded pulse sequences show a significant effect of phase inversion, which is verified to be a consequence of the higher-order effects because such dependence vanishes at the MAS frequency of $100 \mathrm{kHz}$. At the MAS frequency of $100 \mathrm{kHz}$, the ${ }^{13} \mathrm{C}$ recoupling fields were set to $267 \mathrm{kHz}$ and $133 \mathrm{kHz}$ for POST-C $4_{3}^{1}$ and $R 12_{9}^{1}$, respectively. Therefore, the evolution of the spin system must be governed, to a good approximation, by the first-order Hamiltonian, which is not affected by phase inversion. Fig. 2 shows the simulations of the heteronuclear dipolar 
recoupling. At a spinning frequency of $10 \mathrm{kHz}$, the recoupling fields were set to $13.33 \mathrm{kHz}$ and $26.67 \mathrm{kHz}$ for $R 12_{9}^{1}$ and $C 4_{3}^{1}$, respectively. In comparison with the results obtained for a MAS frequency of $100 \mathrm{kHz}$, the higher-order effects are quite significant at long dephasing time. As expected, we find that the phase inversion has no effect on the spin evolution even in the regime of long dephasing time. This dramatic difference in the case of CSA and heteronuclear dipolar recoupling is completely in line with the prediction of our analytical treatment.

\subsection{Experimental verification}

Experimentally we found that the phase convention of our ${ }^{13} \mathrm{C}$ channel is identical to that of the SIMPSON program. The experiment was carried out by adjusting the phase of an rf pulse to spinlock the magnetization evolved under the influence of a predefined offset. Our result is consistent with what reported earlier [11]. CSA recoupling experiments were performed on $\left[1-{ }^{13} \mathrm{C}\right]$-L-alanine. Because the ${ }^{13} \mathrm{C}$ homonuclear dipolar coupling in $\left[1-{ }^{13} \mathrm{C}\right]$-L-alanine
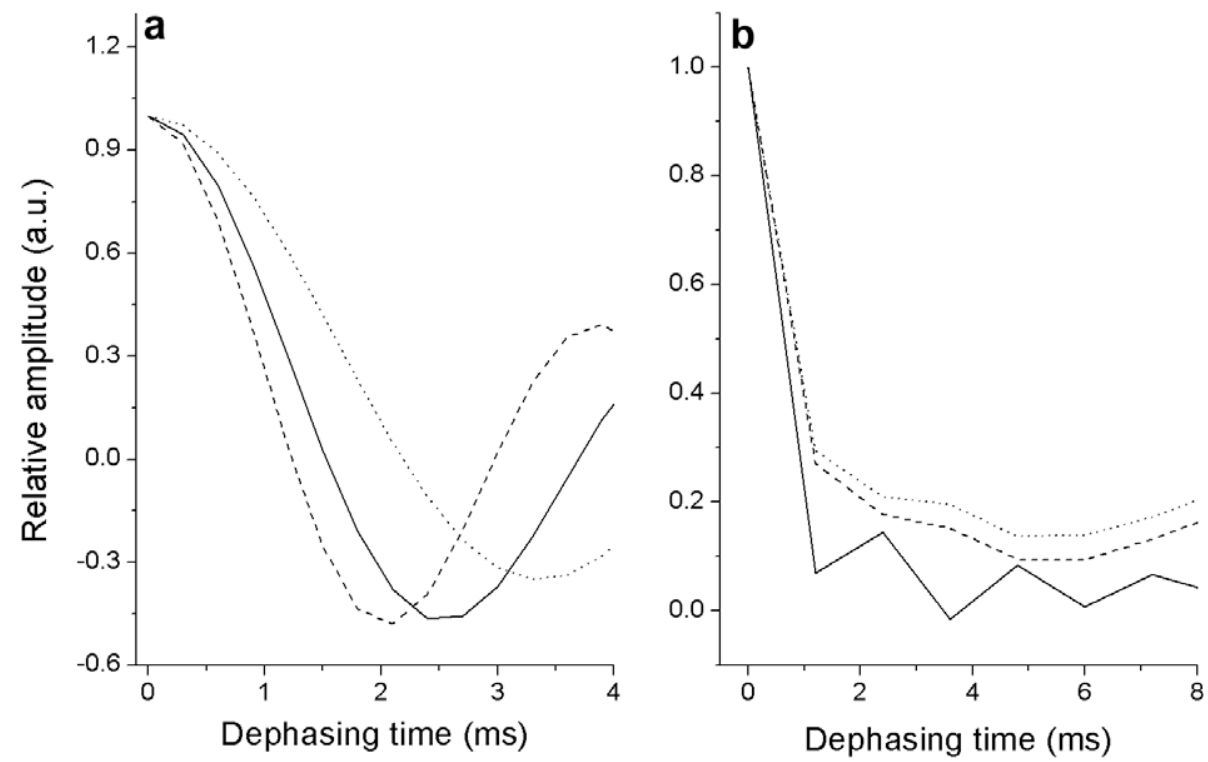

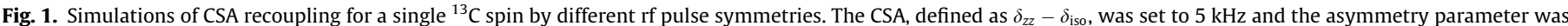

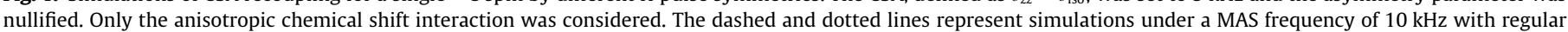

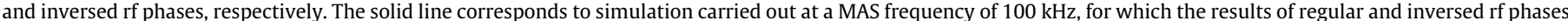

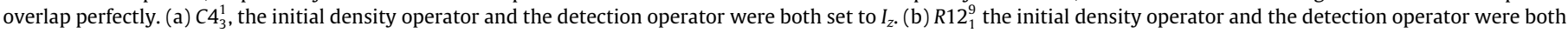
set to $I_{x}$.
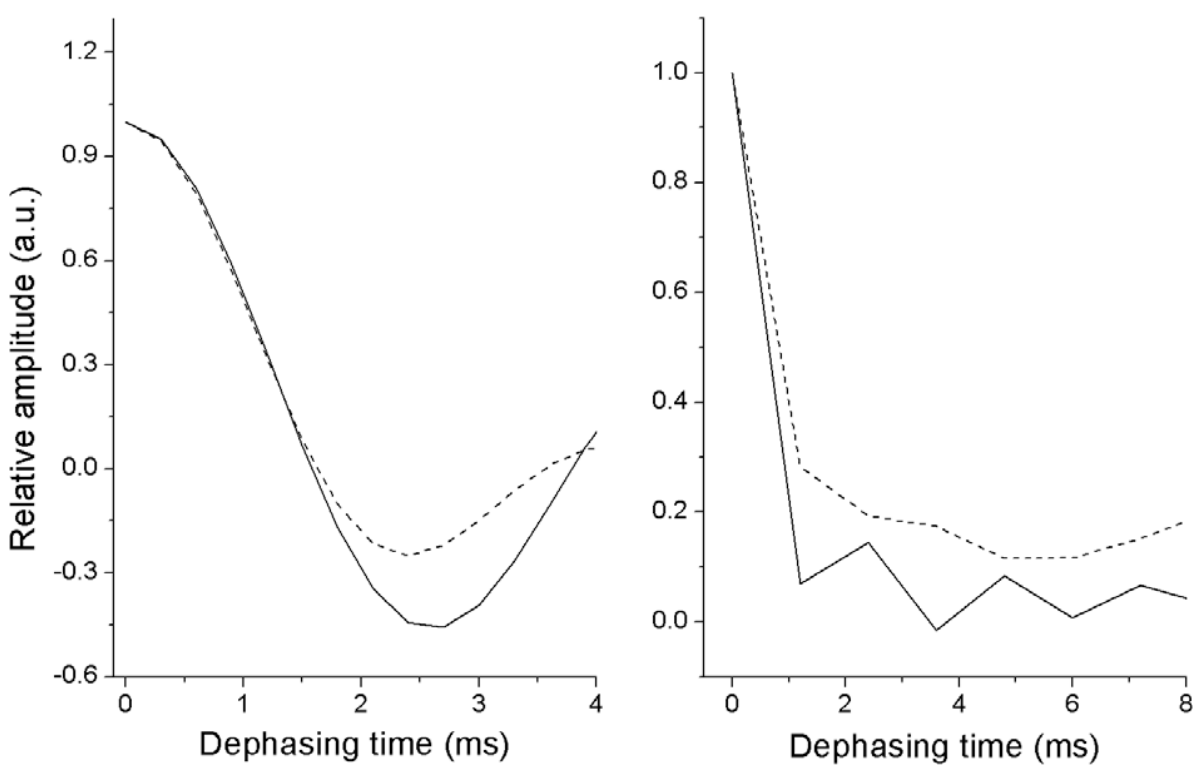

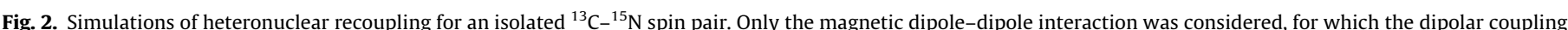

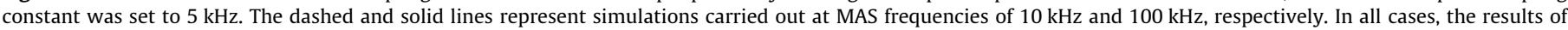
regular and inversed rf phases overlap perfectly. Other simulation conditions are referred to the caption of Fig. 1. 


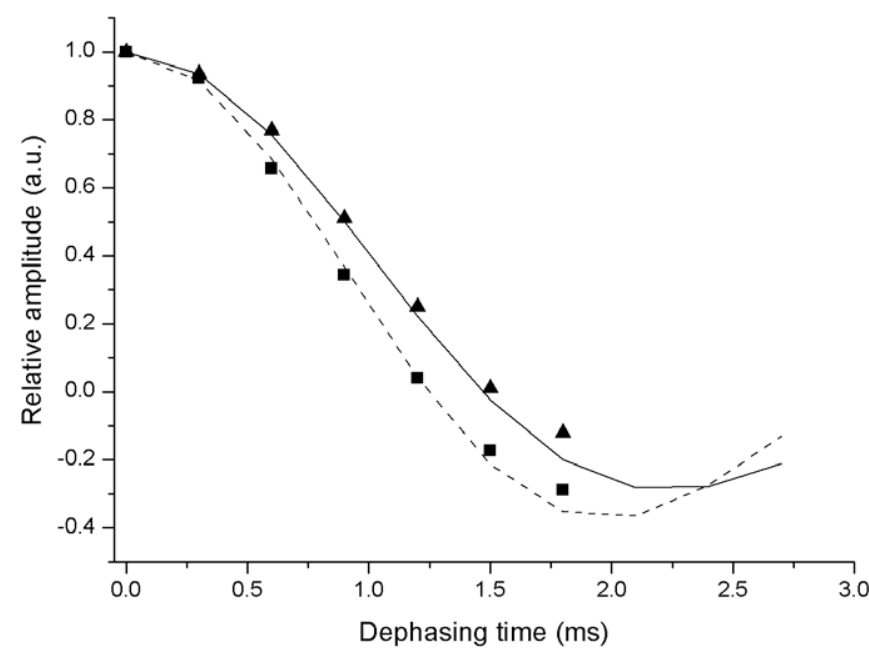

Fig. 3. Experimental and simulation data obtained for $\left[1-{ }^{13} \mathrm{C}\right]-\mathrm{L}$-alanine. Simulation was carried out for a single ${ }^{13} \mathrm{C}$ spin, where the anisotropic chemical shift data were taken from the literature [18]. The triangles and squares represent the experimental data of $\mathrm{C}_{3}{ }_{3}$ with regular and inversed phases, respectively. The solid and dashed lines represent the corresponding simulation results. All the rf field strengths and durations were matched to the experimental conditions and there were no adjustable parameters in the simulations.

is about $102 \mathrm{~Hz}$, which is much weaker than the CSA of carbonyl carbon $(5210 \mathrm{~Hz}$ at 7.05 Tesla), we can neglect the homonuclear dipolar coupling in the data analysis. Fig. 3 shows the experimental and simulation results. Our results highlight again that it is very important to clarify the correspondence between spin-dynamics phases in simulations and pulse program phases of NMR spectrometers, which can, fortunately, be determined experimentally [9$11]$.

\section{Acknowledgments}

This work was supported by grants from the National Science Council and the Ministry of Education. F.-C.C. acknowledges an undergraduate research stipend from NSC. We thank the stimulating comments of the anonymous reviewers.

\section{References}

[1] R. Tycko, Prog. Nucl. Magn. Reson. Spectrosc. 42 (2003) 53.

[2] M. Baldus, Prog. Nucl. Magn. Reson. Spectrosc. 41 (2002) 1.

[3] S. Luca, H. Heise, M. Baldus, Acc. Chem. Res. 36 (2003) 858.

[4] M.H. Levitt, Symmetry-based pulse sequence in magic-angle spinning solidstate NMR, in: D.M. Grant, R.K. Harris (Eds.), Encyclopedia in Nuclear Magnetic Resonance, Wiley, Chichester, 2002, p. 165.

[5] M.H. Levitt, J. Chem. Phys. 128 (2008) 052205.

[6] J.C.C. Chan, Chem. Phys. Lett. 335 (2001) 289.

[7] J.C.C. Chan, H. Eckert, J. Chem. Phys. 115 (2001) 6095.

[8] P.E. Kristiansen, M. Carravetta, J.D. van Beek, W.C. Lai, M.H. Levitt, J. Chem Phys. 124 (2006) 234510.

[9] M.H. Levitt, J. Magn. Reson. 126 (1997) 164.

[10] M.H. Levitt, O.G. Johannessen, J. Magn. Reson. 142 (2000) 190.

[11] M.H.A. Roehrl, G.J. Heffron, G. Wagner, J. Magn. Reson. 174 (2005) 325.

[12] S. Hediger, B.H. Meier, N.D. Kurur, G. Bodenhausen, R.R. Ernst, Chem. Phys. Lett. 223 (1994) 283.

[13] S. Hediger, B.H. Meier, R.R. Ernst, Chem. Phys. Lett. 240 (1995) 449.

[14] A. Detken, E.H. Hardy, M. Ernst, B.H. Meier, Chem. Phys. Lett. 356 (2002) 298.

[15] M. Hohwy, H.J. Jakobsen, M. Eden, M.H. Levitt, N.C. Nielsen, J. Chem. Phys. 108 (1998) 2686.

[16] M. Bak, J.T. Rasmussen, N.C. Nielsen, J. Magn. Reson. 147 (2000) 296.

[17] M. Bak, N.C. Nielsen, J. Magn. Reson. 125 (1997) 132.

[18] A. Naito, S. Ganapathy, K. Akasaka, C.A. McDowell, J. Chem. Phys. 74 (1981) 3190. 